\title{
Folic acid-functionalized drug delivery platform of resveratrol based on Pluronic I27/D- $\alpha$-tocopheryl polyethylene glycol 1000 succinate mixed micelles
}

This article was published in the following Dove Press journal:

International Journal of Nanomedicine

24 March 2017

Number of times this article has been viewed

\author{
Jifu Hao* \\ Tiantian Tong* \\ Kai Jin \\ Qiannan Zhuang \\ Te Han \\ Yanping Bi \\ Jianzhu Wang \\ Xiaodan Wang \\ College of Pharmacy, Taishan \\ Medical University, Taian, People's \\ Republic of China \\ *These authors contributed equally \\ to this work
}

Correspondence: Jifu Hao;

Xiaodan Wang

College of Pharmacy, Taishan Medical

University, No 619, Changcheng Road,

Taian 270016, People's Republic of China

Tel +86538622 975 I

Email haojifu@।63.com;

xdwang@tsmc.edu.cn

\begin{abstract}
A folic acid (FA)-functionalized drug vehicle platform based on Pluronic 127 (P127)/D- $\alpha$-tocopheryl polyethylene glycol 1000 succinate (TPGS) mixed micelles was orchestrated for an effective delivery of the model drug resveratrol in order to address the problem of poor water solubility and rapid metabolism of resveratrol and improve its targeted accumulation at tumor site. The FA-decorated mixed micelles were prepared using thin-film hydration method and optimized by central composite design approach. The micelles were also characterized in terms of size and morphology, drug entrapment efficiency and in vitro release profile. In addition, the cytotoxicity and cell uptake of the micelles were evaluated in folate receptor-overexpressing MCF-7 cell line. In vivo pharmacokinetic and biodistribution studies were also performed. The average size of the micelles was $\sim 20 \mathrm{~nm}$ with a spherical shape and high encapsulation efficiency (99.67\%). The results of fluorescence microscopy confirmed the targeting capability of FA-conjugated micelles in MCF-7 cells. FA-modified micelles exhibited superior pharmacokinetics in comparison with that of solution. Further, the low accumulation of resveratrol-loaded FA micelles formulation in the heart and kidney avoided toxicity of these vital organs. It could be concluded that folate-modified P127/TPGS mixed micelles might serve as a potential delivery platform for resveratrol.
\end{abstract}

Keywords: resveratrol, Pluronic 127/TPGS, mixed micelles, folic acid conjugation

\section{Introduction}

In recent years, resveratrol, a naturally occurring polyphenol and phytoalexin, abundant in berry, grape, peanut and other plants, has sparked significant focus due to its beneficial effects on human health, which include antioxidant, anti-inflammatory, cardioprotective and antitumor activities. ${ }^{1}$ Some literature have illustrated that resveratrol can both inhibit tumor cell growth and promote cell apoptosis exerting its antitumor effect by means of some related cell signaling pathways. ${ }^{2,3}$ However, poor bioavailability of resveratrol owing to factors such as low aqueous solubility, easy photodegradation and extensive first-pass metabolism hinders its further clinical application. ${ }^{4}$ Therefore, the primary problem is how to design effective and suitable drug delivery systems (DDSs) for resveratrol to address the aforementioned shortcomings. Innovative DDSs of resveratrol, such as polymeric nanoparticles, cyclodextrins, colloidal mesoporous silica nanoparticles and liposomes, have been fabricated to enhance aqueous solubility and bioavailability, improve physicochemical stability and achieve targeted or controlled drug release. ${ }^{5-7}$ Recently, different classical and successful nanotherapeutics used to transport anticancer drugs into tumors have been fabricated and applied in order to improve the anticancer effect of chemotherapeutic 
drugs, the most prominent among which include polymeric nanocarriers such as liposomes, supramolecular derivatives and ultradeformable vesicles. ${ }^{8-10}$ As one of the promising nanoparticular approaches, the mixed micelle carriers formed by triblock copolymers for delivery of anticancer drugs have been basically exploited in various clinical applications for the merits of smaller size, self-assembling ability, solubilization and protection against unstable drugs. ${ }^{11}$

Nonetheless, many anticancer drugs incorporated into micelles show rare selectivity and limited orientation for malignant tumors because of their passive targeting efficiency described by the well-known enhanced permeability and retention (EPR) effect. These passive DDSs have exhibited distinctly nonspecific distribution in systemic administration and resulted in toxicity against normal tissues. Selective and preferred DDSs intended to specific sites will not only improve therapeutic efficacy but also avoid negative side effects on normal cells and tissues. ${ }^{12}$ Accordingly, it is necessary to make use of the specific receptors present on the surface of cell membrane to achieve better tumor targeting. Some strategies of conjugation of targeting ligand molecules, such as folic acid (FA), mannose, RGD peptide and transferrin, to block polymers for selective delivery, have been reported in published literature. ${ }^{13}$

The folic acid receptor (FAR) has been found to be overexpressed on the surface of some human solid tumors. Hence, FA is easily internalized into cells if it encounters with FAR present on the surface of tumor cells via the well-known receptor-mediated endocytosis mechanism. Therefore, FA is a common choice as a targeting ligand molecule due to its many benefits, such as nonimmunogenicity, small size, nontoxicity and ease of handling, and can be used as a targeting moiety to mediate various anticancer agents into targeted cells to evade their nonspecific attacks on normal tissues. ${ }^{14,15}$

However, in order to further increase the effectiveness of active targeting modalities, long circulation time in vivo is an essential prerequisite to allow functionally active targeting nanoparticles to pursue more opportunities to bind to the receptors present on the surface of the cell membranes. This prolongation in circulation time can be achieved by Pluronic 127 (P127) triblock copolymer micelle nanoparticles, which have been studied for many years in applications ranging from drug to gene carriers. ${ }^{16}$ The core-shell configuration of mixed micelles is beneficial to increasing drug stability and solubility as well as providing a prolonged circulation period and preferential accumulation in tumors via the EPR effect after administration. ${ }^{17,18}$
Therefore, considering the advantage of resveratrol as a potential active ingredient and utilizing mixed micelles as a suitable nanoparticulate carrier, in the present study, we orchestrated a mixed micelles system loading resveratrol for its antitumor activity and FA-functionalized P127 for folatemediated receptor targeting to cancer cells. FA-functionalized P127 was fabricated by the traditional esterification reaction. The properties of FA-conjugated P127 were also evaluated by proton nuclear magnetic resonance ( $\left.{ }^{1} \mathrm{HNMR}\right)$ and infrared (IR) spectrum. The mixed micelles were prepared by thin-film dispersion method, and the formulation was optimized with the central composite design (CCD) response surface method. In addition, the physicochemical properties, antitumor efficacy in vitro with the uptake by MCF-7 tumor cells and pharmacokinetics and biodistribution of mixed micelles were investigated.

\section{Materials and methods Materials and animals}

Commercial trans-resveratrol (98.9\% pure) was obtained from Tianjin Jianfeng Natural Product R\&D Co., Ltd. (Tianjin, People's Republic of China). D- $\alpha$-Tocopheryl polyethylene glycol 1000 succinate (TPGS) was purchased from SigmaAldrich (St Louis, MO, USA). 1,1-Carbonyldiimidazole (CDI), N-hydroxysuccinimide (NHS) and 1,3-dicyclohexylcarbodiimide (DCC) were obtained from Aladdin Reagent Co., Ltd. (Shanghai, People's Republic of China). Poloxamer 127 (ie, P127) was kindly supplied by BASF (China) Co., Ltd. (Shanghai, People's Republic of China). Acetonitrile of high-performance liquid chromatography (HPLC) grade was used, and all other reagents and solvents used were of analytical reagent grade.

Sprague Dawley rats (200-220 g) and Kunming strain mice (18-22 g) were obtained from the Lukang Animal Center (Jining, People's Republic of China). All animals were handled according to the Principles of Laboratory Animal Care, and the protocols were approved by the Taishan Medical University Animal Ethical Committee.

\section{Synthesis and characterization of FA- decorated PI27}

The concrete synthetic methods of conjugation of FA with P127 were performed as described earlier by Zhang et $\mathrm{a}^{19}$ and are shown in Figure 1. First, in order to activate P127, $12.6 \mathrm{~g}$ of P127 was dissolved in $15 \mathrm{~mL}$ of anhydrous acetonitrile. This solution was introduced dropwise into a CDI (1.62 g) anhydrous acetonitrile solution at room temperature for $2 \mathrm{~h}$ under nitrogen atmosphere, and then the homogeneous mixture was stirred overnight under similar conditions. After 


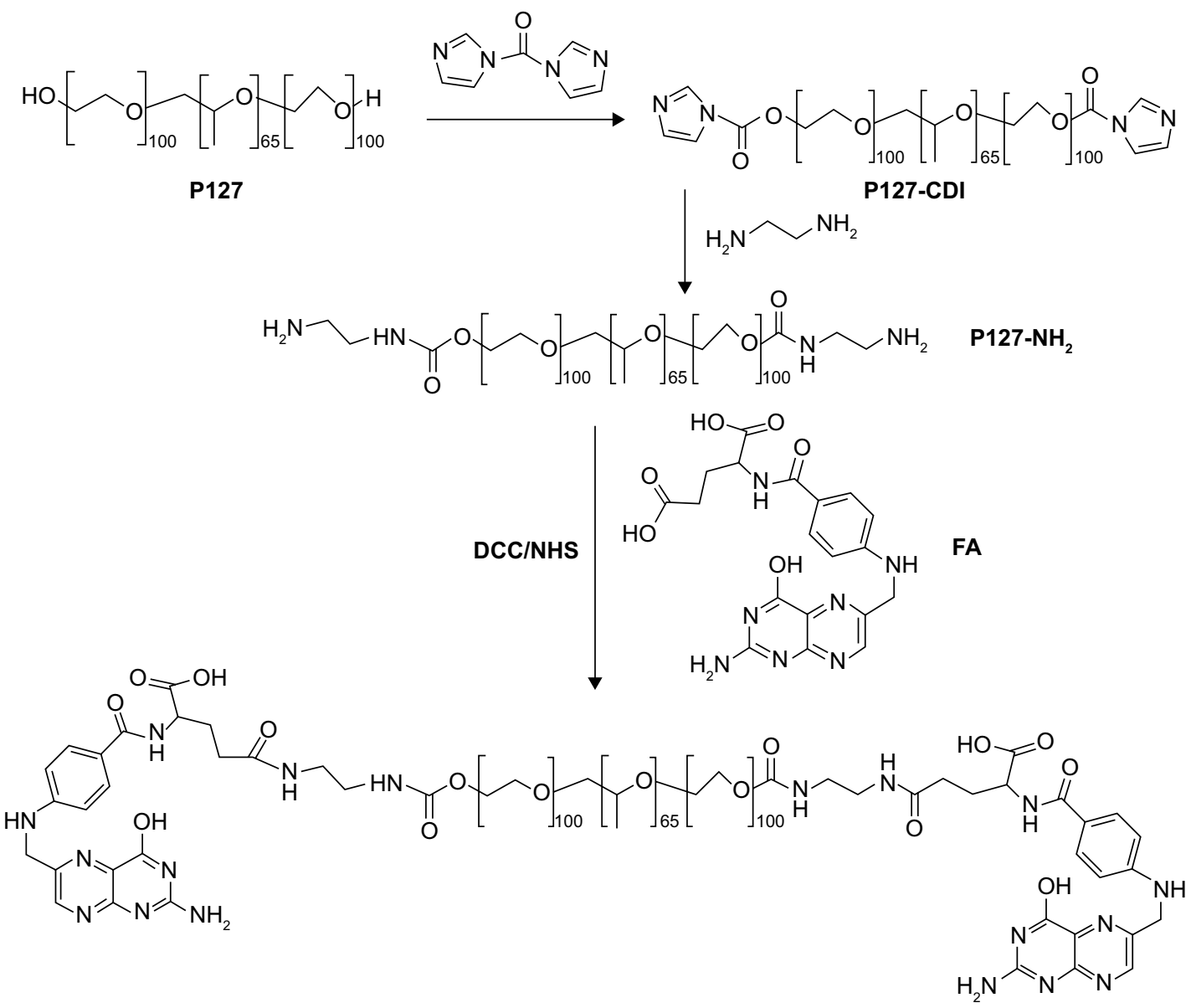

FA-P127

Figure I Schematic illustration of the synthesis routes of FA-PI27.

Abbreviations: PI27, Pluronic 127; CDI, I, I-carbonyldiimidazole; DCC, I,3-dicyclohexyl-carbodiimide; NHS, N-hydroxysuccinimide; FA, folic acid.

concentration, the unreacted CDI was removed by precipitation with excessive ethyl ether. The resulting precipitate was collected by filtration and dried by vacuum dehydration to obtain CDI-activated P127.

The prefabrication of amino-terminated $\mathrm{P} 127$ was achieved via the reaction of CDI-P127 with 1,2-ethylenediamine in acetonitrile solution at room temperature for $2 \mathrm{~h}$, and the mixture was allowed to react overnight. After removal of redundant and unreacted ethylenediamine, the $\mathrm{P} 127-\mathrm{NH}_{2}$ product was purified using excess amount of ether, and a white precipitate was obtained.

Finally, in order to tailor folate-decorated P127 (FA-P127), the P127- $\mathrm{NH}_{2}$ (1 g, $\left.0.079 \mathrm{mmol}\right), \mathrm{FA}(105 \mathrm{mg}$, $0.237 \mathrm{mmol})$, NHS (60 mg, $0.522 \mathrm{mmol})$ and DCC (108 mg, $0.522 \mathrm{mmol}$ ) were codissolved in $10 \mathrm{~mL}$ dimethyl sulfoxide (DMSO). This reaction mixture was kept in a nitrogen atmosphere at room temperature in the dark and stirred overnight using triethylamine as a catalyzer for the reaction to get completed. Then, after isolation of by-product dicyclohexylurea, the supernatant was dialyzed against deionized water for 2 days. The obtained P127-FA was freeze-dried and then confirmed by ultraviolet (UV), IR and ${ }^{1} \mathrm{H}$ NMR analyses. The full absorption spectrum of P127-FA was scanned from 200 to $400 \mathrm{~nm}$ in phosphate buffer saline (PBS; $\mathrm{pH} 7.4$ ) with a UV vision spectrophotometer (UV-2550; Shimadzu, Kyoto, Japan) to confirm whether the grafted FA was present on the block polymers' skeleton or not. After grinding the various samples with $\mathrm{KBr}$, transparent $\mathrm{KBr}$ tablets were prepared and used to obtain Fourier transform infrared (FTIR) spectra (LabSolutions IR; Shimadzu). ${ }^{1} \mathrm{H}$ NMR analysis results were recorded on nuclear magnetic resonance spectrometer (Avance $400 \mathrm{MHz}$; Bruker, Billerica, MA, USA) using deuterium chloroform $\left(\mathrm{CDCl}_{3}\right)$ as a solvent.

\section{Preparation of resveratrol-loaded mixed micelles}

The preparation of resveratrol-loaded polymeric mixed micelles was achieved via thin-film hydration method. ${ }^{20}$ 
Table I Variables and their levels in the CCD

\begin{tabular}{llll}
\hline Independent variables & \multicolumn{2}{l}{ Levels } & \\
\cline { 2 - 4 } & $-\mathbf{I}$ & $\mathbf{0}$ & $\mathbf{I}$ \\
\hline$A=$ dosage of resveratrol $(\mathrm{mg} / \mathrm{mL})$ & 0.25 & 0.625 & $\mathrm{I}$ \\
$B=$ mass ratio of $\mathrm{PI} 27$ and TPGS & $2: 5$ & $7: 5$ & $\mathrm{I} 2: 5$ \\
Dependent variables & Constraints & \\
$Y_{1}=$ entrapment efficiency $(\%)$ & Maximize & \\
$Y_{2}=$ drug loading $(\%)$ & Maximize & \\
$Y_{3}=$ particle size $(\mathrm{nm})$ & Minimize & \\
\hline
\end{tabular}

Abbreviations: CCD, central composite design; PI27, Pluronic 127; TPGS, D- $\alpha$ tocopheryl polyethylene glycol 1000 succinate.

Briefly, the mixture with a fixed mass ratio $(1: 10)$ of resveratrol and polymer carriers (the different proportions of FA-P127 and TPGS are shown in Table 1) was codissolved in $10 \mathrm{~mL}$ ethanol. Subsequently, ethanol was removed by evaporating at $40^{\circ} \mathrm{C}$ under vacuum to develop uniform thin film. The resultant film was hydrated at room temperature by ultrasonic treatment to obtain a clear micelles solution, which was then centrifuged at $13,204 \mathrm{~g}$ for $15 \mathrm{~min}$ to separate unloaded drugs. The similar protocol was adopted to prepare empty mixed micelles and fluorescein isothiocyanate (FITC)-loaded micelles except for incorporation of model drug.

\section{Optimization of mixed micelles by CCD}

After preliminary screening studies, two variables, that is, the amount of resveratrol $(A)$ and the mass ratio of $\mathrm{P} 127$ and TPGS $(B)$ in the mixed micelles, were chosen to optimize formulation using dependent responses including drug loading (DL), entrapment efficiency (EE) and particle size as criteria. The experimental schedule based on CCD response surface method is described in Table 1.

\section{Characterization of resveratrol mixed micelles}

Particle size and transmission electron microscopy (TEM)

Particle size and size distribution were measured by Malvern Zetasizer Nano-ZS (Malvern Instruments Ltd., Malvern, UK) at $25^{\circ} \mathrm{C}$. Measurements were performed in triplicate for every prepared sample. The morphological profile of resveratrol mixed micelles was determined by TEM (JEM-1200EX; JEOL, Tokyo, Japan). A drop of micellar solution was loaded onto a copper grid, and then negatively stained with phosphotungstic acid solution (2\%, $\mathrm{w} / \mathrm{v}$ ) for a while. After getting rid of excess solution with filter papers, samples were dried appropriately and then examined under TEM.

\section{Determination of $\mathrm{DL}$ and $\mathrm{EE}$}

The content of resveratrol encapsulated in mixed micelles was determined by UV-Vis spectrophotometer at $306 \mathrm{~nm}$. The prepared micellar solution was diluted to a certain concentration with acetonitrile before determination. The DL\% and $\mathrm{EE} \%$ of resveratrol in polymeric micelles were calculated from the following equations: ${ }^{21}$

$$
\begin{gathered}
\mathrm{DL} \%=\frac{\text { Weight of the drug in micelles }}{\text { Weight of the feeding polymer and drug }} \times 100 \\
\mathrm{EE} \%=\frac{\text { Weight of the drug in micelles }}{\text { Weight of the feeding drug }} \times 100
\end{gathered}
$$

\section{Critical micelle concentration (CMC) determination}

The CMC of mixed micelles can be determined by evaluation of pyrene absorption in surfactant solution. ${ }^{22}$ Once the micelles are formed, pyrene would transfer into micellar hydrophobic cores from the aqueous phase, which will lead to an alteration in the intensity ratio of I372/I383. Briefly, $100 \mu \mathrm{L}$ of pyrene acetone solution $\left(6 \times 10^{-5} \mathrm{M}\right)$ was introduced into an amber glass bottle, and acetone was removed under flow of nitrogen. Ten milliliters of P127 and TPGS micelles with a concentration range from $2.4448 \times 10^{-4}$ to $1.001 \mathrm{mg} /$ $\mathrm{mL}$ were mixed with the residue by ultrasonic treatment for $30 \mathrm{~min}$. The mixture was incubated in the absence of light overnight for equilibrium. Fluorescence intensities at an emission wavelength of $372 \mathrm{~nm}$ (I372) and $383 \mathrm{~nm}$ (I383) were measured, and their ratio (I372/I383) was plotted against the concentration of micelles. The graph resulting from I372/I383 fluorescence intensity ratio versus logarithm of concentration was analyzed according to the following Boltzmann sigmoidal equation to evaluate the $\mathrm{CMC}$ of the studied surfactants. ${ }^{22}$

$$
Y=\frac{A_{1}-A_{2}}{1+\mathrm{e}^{\left(x-x_{0}\right) / \Delta x}}+A_{2}
$$

where $Y$ represents the ratio value of I372/I383, $\mathrm{x}$ means the concentration of mixed surfactant, $A_{1}$ and $A_{2}$ stand, respectively, for the initial and final asymptotes of sigmoid, $x_{0}$ is regarded as the center of sigmoid and $\Delta x$ is connected with the independent variable occurrence.

\section{In vitro drug release}

In vitro release behaviors of resveratrol from the mixed micelles were evaluated by dialysis (molecular weight cutoff $30 \mathrm{kDa}$ ) method. In order to fit the sink condition, PBS 
(pH 7.4) with $0.5 \%$ Tween 80 was adopted as the release medium. Two milliliters of resveratrol mixed micelles or $2 \mathrm{~mL}$ resveratrol solution (in propylene glycol) as control was sealed into a preswollen dialysis bag. Then, the dialysis pockets were immersed into $50 \mathrm{~mL}$ release medium at $37^{\circ} \mathrm{C}$ at a stirring speed of $120 \mathrm{rpm}$. At each presetting time interval, $1 \mathrm{~mL}$ aliquots of release medium were withdrawn, and the same volume of fresh medium was supplied. After centrifugation at 13,204 $\mathrm{g}$ for $15 \mathrm{~min}$, the supernatant drug content was determined by HPLC at $306 \mathrm{~nm}$. The HPLC system comprised an LC-10A pump (Shimadzu) with a UVVis detector. A Thermo Fisher Scientific C18 (250×4.6 mm) analytical column was used with the mobile phase made of acetonitrile and $0.1 \%(\mathrm{v} / \mathrm{v})$ aqueous phosphoric acid (30:70). The flow rate was $1.0 \mathrm{~mL} / \mathrm{min}$ at room temperature. Then, the cumulative release percentage $(Q \%)$ was calculated.

\section{In vitro cell evaluation Cytotoxicity assay}

The in vitro cytotoxicity of micelles loaded with drug was evaluated in MCF-7 cells using CCK-8 method. MCF-7 cell lines were purchased commercially from Cell Resource Center of the Chinese Academy of Sciences (Shanghai, People's Republic of China). The cells were cultured in 96-well microtiter plate with routine RPMI 1640 medium containing $10 \%$ fetal bovine serum at $37^{\circ} \mathrm{C}$ in a humidified $5 \% \mathrm{CO}_{2}$ sterile incubator. Until the cell density reached $\sim 2 \times 10^{4}$ cells per well, the culture medium was replaced by $200 \mu \mathrm{L}$ of fresh medium containing the following treatment agents: resveratrol DMSO solution (the final concentration of DMSO should be $<0.2 \%$ ), resveratrol-loaded micelles and FA-modified resveratrol micelles. The concentration of resveratrol ranged from 0 to $300 \mu \mathrm{g} / \mathrm{mL}$. After further $48-\mathrm{h}$ incubation, the cells were washed twice with PBS, and then the cell viability was determined using the CCK-8 assay in light of standard protocol.

\section{Cell uptake study}

For evaluation of the targeting potential of developed delivery systems in vitro, cell uptake studies of FA-mixed micelles were performed in folate receptor-overexpressing $\mathrm{MCF}-7$ cells. FITC, a hydrophobic fluorescent probe, was encapsulated into a lipophilic core of mixed micelles as a tracer. MCF-7 cells were seeded in 24 plates at $2 \times 10^{4}$ cells per well with folate-free Dulbecco's Modified Eagle's Medium (DMEM) to allow attachment for $24 \mathrm{~h}$. After the cells reached $80 \%$ confluence, the primary medium was replaced with fresh medium containing fluorescent FITC micelles, and then the cells were further incubated for $2 \mathrm{~h}$. Subsequently, the supernate was withdrawn, and the cells were washed with PBS thrice to remove the plethora of micelles adsorbed on the cell surface and observed under the fluorescence microscope. For free-folate competition studies, the cells were previously incubated for $30 \mathrm{~min}$ in the presence of $1 \mathrm{mM} \mathrm{FA}$-containing DMEM and then treated with the drug-loaded polymeric micelles. Then, the cells were collected with $0.5 \mathrm{~mL}$ PBS and subjected to ultrasonic treatment (100 w, $2 \mathrm{~min}$ ) in ice bath. The fluorescence intensity of FITC was measured with a microplate reader at an excitation wavelength of $488 \mathrm{~nm}$ and an emission wavelength of $525 \mathrm{~nm}$.

\section{In vivo animal studies}

\section{Pharmacokinetic studies}

Pharmacokinetic studies of resveratrol-loaded micelles compared with resveratrol solution were carried out. Ten Sprague Dawley rats, weighting 220-250 g, were randomly divided into two groups before the experiments. The experiment procedures were approved by the ethics committee of laboratory animal center of Taishan Medical University. After administration of the same dosage of resveratrol solution or resveratrol micelles $(23.1 \mathrm{mg} / \mathrm{kg}$, expressed as resveratrol equivalents) through tail vein, the whole-blood sample $(\sim 0.5 \mathrm{~mL})$ was collected into a heparinized tube by sinus jugularis puncture from each rat in every group at 0.25 , $0.5,1,2,3,4,6$ and $8 \mathrm{~h}$, respectively. Blood samples were centrifuged for $10 \mathrm{~min}$ at 4,000 rpm, and then the plasma was collected and stored at $-80^{\circ} \mathrm{C}$.

\section{Tissue distribution studies}

The Kunming strain mice were divided into two groups at random and treated with resveratrol micelles or resveratrol solution via tail vein injection at a dose of $10 \mathrm{mg} / \mathrm{kg}$ to mice, respectively. At predetermined time, the blood of mice was collected from postorbital vein into heparinized tubes and then centrifuged to get the plasma samples. Tissues, such as the heart, liver, spleen, lung and kidney, were harvested immediately after heart perfusion with PBS, and the redundant water in the isolated organ was removed with filter paper. The plasma and tissue samples were stored at $-80^{\circ} \mathrm{C}$ until analysis.

\section{Plasma and tissue sample analysis}

One hundred microliters of plasma samples was blended with $300 \mu \mathrm{L}$ acetonitrile (containing chlorzoxazone as internal standard) in a tube to precipitate protein, vortexed for $3 \mathrm{~min}$ and centrifuged for $10 \mathrm{~min}$ at $12,000 \mathrm{rpm}$ at $4{ }^{\circ} \mathrm{C}$, and then 
the supernate was transferred to another new tube and dried under nitrogen gas stream at room temperature. The residue was dissolved in $100 \mu \mathrm{L}$ acetonitrile, after vortexing and centrifugation for $10 \mathrm{~min}$, and the supernate sample was used for HPLC analysis. Tissue sample was weighted accurately and homogenized after addition of moderate PBS to obtain $1 \mathrm{mg} / \mathrm{mL}$ homogenate. Treatment of tissue homogenates was done using the same method as plasma samples, and the samples were analyzed by HPLC.

The plasma concentration-versus-time profile was analyzed by DAS Version 2.0. Various pharmacokinetic parameters were calculated such as the area under the plasma concentration-time curve from zero to infinity $\left(\mathrm{AUC}_{0 \rightarrow \infty}\right)$ and mean residence time (MRT). All data were expressed as the mean \pm standard deviation (SD).

\section{Results and discussion Synthesis and characterization of FA-PI 27}

The functional copolymer FA-P127 with the targeting property was synthesized by the esterification reaction mediating conjugation of FA with P127. The synthetic routes are illustrated in Figure 1. The chemical structures of the copolymers were characterized by ${ }^{1} \mathrm{H}$ NMR (100 MHZ, DMSO-d ${ }_{6}$, ppm) and FTIR.

As shown in Figure 2A, the results of ${ }^{1} \mathrm{H}$ NMR disclosed that the peaks at $\delta 1.05 \mathrm{ppm}\left(\mathrm{d}, 3 \mathrm{H},-\mathrm{CH}_{3}\right.$ of polypropylene oxide [PPO] ) and 3.36-3.50 ppm (m, 3H, 4H, $-\mathrm{CH}_{2} \mathrm{CHO}-$ of PPO and $-\mathrm{CH}_{2} \mathrm{CH}_{2} \mathrm{O}-$ of polyethylene oxide [PEO]) were attributed to the protons of PEO and PPO groups in P127. While the new characteristic peaks emerged from protons in the FA structure, such as at $\delta 4.45$ and 8.65 ppm, which were, respectively, related to $\mathrm{C}_{9}-\mathrm{H}$ and $\mathrm{C}_{7}-\mathrm{H}$ of $\mathrm{FA}$, peaks at $\delta 6.65$ and $7.62 \mathrm{ppm}$ belonged to aromatic protons of the phenyl groups of FA. ${ }^{23}$ In addition, as seen from FTIR spectra in Figure 2, typical C-H stretching vibrations of $\mathrm{PEO}$ segments appeared at 3,001 and $2,887 \mathrm{~cm}^{-1}$. The peak at $1,112 \mathrm{~cm}^{-1}$ was attributed to the characteristic $\mathrm{C}-\mathrm{O}-\mathrm{C}$ stretching vibration in $\mathrm{P} 127$ (Figure 2B). The peaks in the range of 1,484-1,697 $\mathrm{cm}^{-1}$ were ascribed to the aromatic ring stretch in the pyridine and benzene ring of FA (Figure 2C). The absorption peaks that occurred between 1,609 and $1,718 \mathrm{~cm}^{-1}$ (Figure 2D) belonged to the amide group (-CO-NH-) in the $\mathrm{P} 127-\mathrm{FA}$ structure. In general, these results of the ${ }^{1} \mathrm{H}$ NMR and FT IR spectra confirmed the presence of FA in the FA-P127 grafting copolymers. ${ }^{24}$ To further demonstrate

A
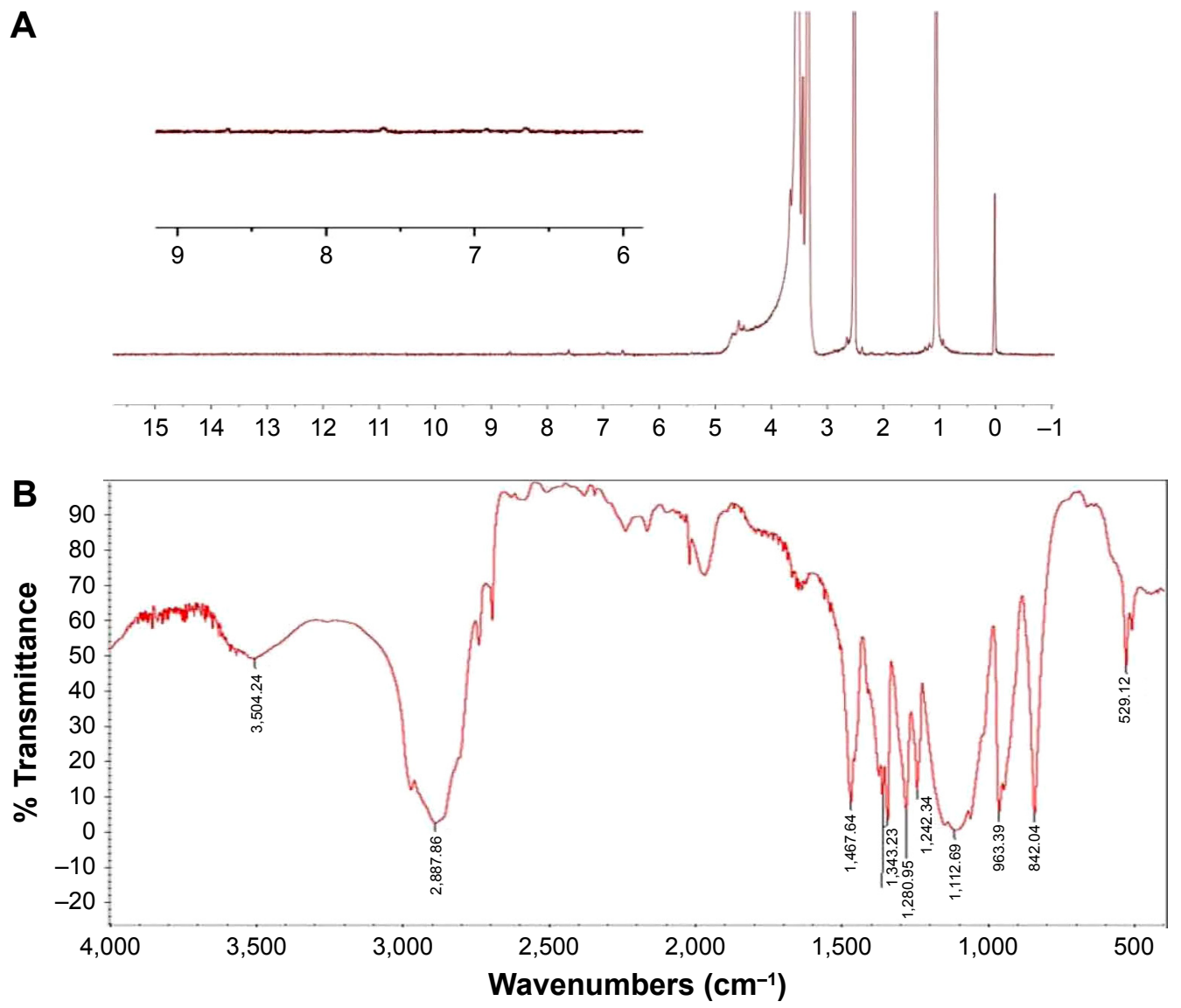

Figure 2 (Continued) 

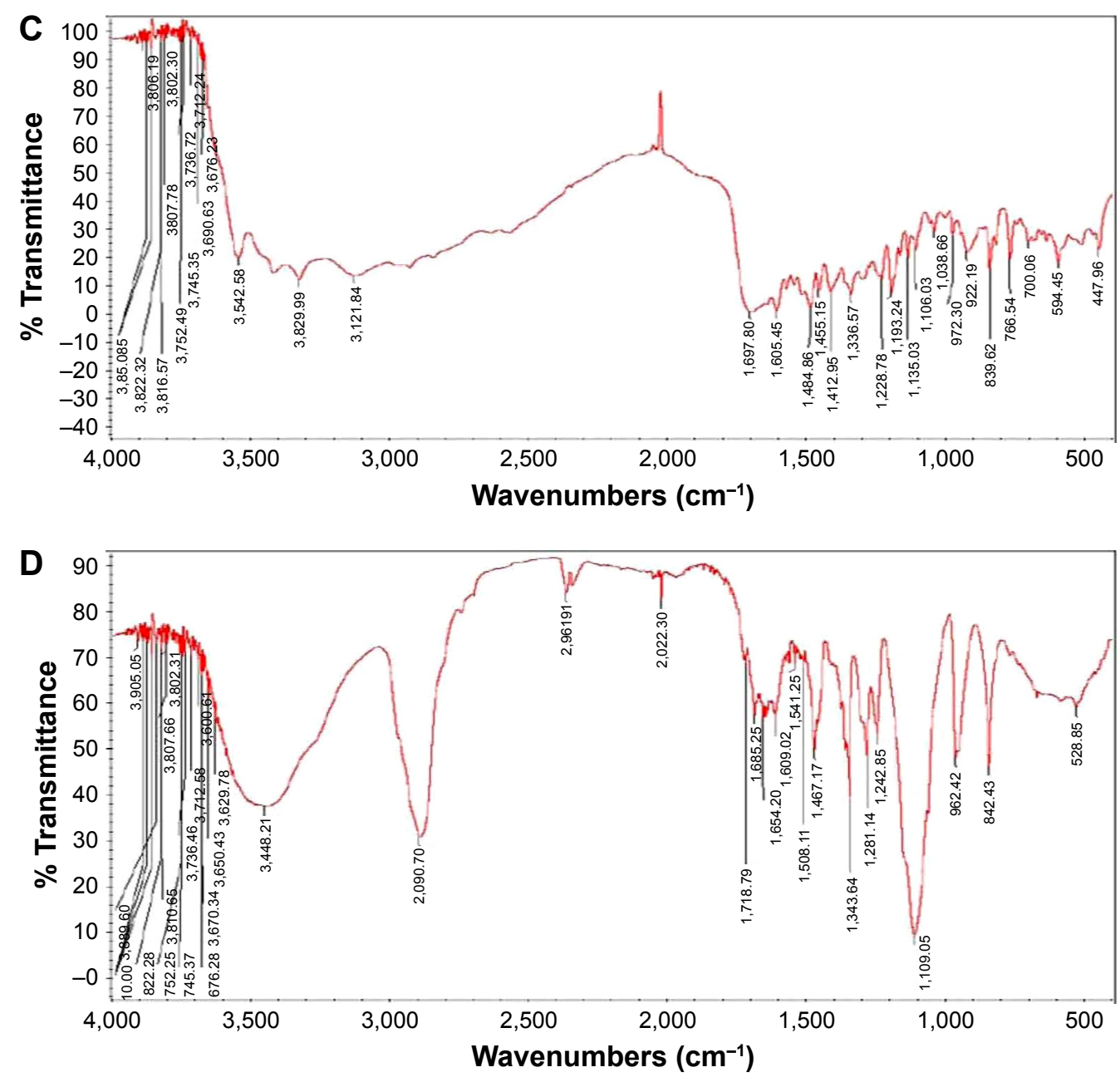

E

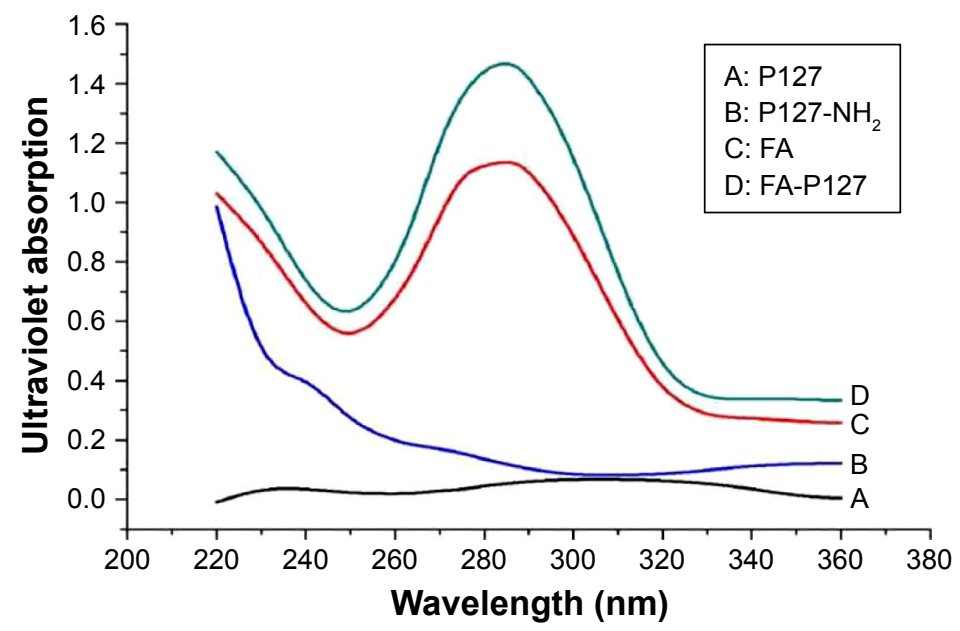

Figure 2 The physicochemical characteristics of functional FA-PI27: 'H NMR spectrum of FA-PI27 (A); IR spectrum of PI27 (B); IR spectrum of FA (C); FTIR spectrum of FA-PI 27 (D); and UV spectrum of FA, PI27 and FA-PI27 (E).

Abbreviations: FA, folic acid; PI27, Pluronic I27; 'H NMR, proton nuclear magnetic resonance; IR, infrared; FTIR, Fourier transform infrared; UV, ultraviolet.

whether the synthesis of P127-FA is successful or not, UV spectrum analysis was also carried out at each step of the synthesis. In Figure 2E, the similar peaks that appeared at wavelength $280 \mathrm{~nm}$ were regarded as those characteristic of FA due to the aromatic ring in P127-FA. ${ }^{25}$

\section{Optimization of resveratrol-loaded} micelles based on CCD

The detailed experimental results obtained using CCD are listed in Table 2, and the results allowed to generate regression equations after thorough statistical analysis. All the 
Table 2 Experimental runs and results of resveratrol micelles based on CCD

\begin{tabular}{llllll}
\hline Formulae & RES $(\mathbf{m g} / \mathbf{m L})$ & P I 27/TPGS & EE\% & DL\% & PS (nm) \\
\hline MI & 0.625 & $7: 5$ & 97.90 & 8.96 & 20.01 \\
& 0.625 & $7: 5$ & 95.41 & 8.72 & 20.37 \\
& 0.625 & $7: 5$ & 97.61 & 9.08 & 21.38 \\
& 0.625 & $7: 5$ & 98.65 & 9.01 & 19.90 \\
M2 & I & $2: 5$ & 72.88 & 6.64 & 24.24 \\
M3 & 0.625 & $2: 5$ & 92.27 & 8.40 & 24.97 \\
M4 & 0.25 & $2: 5$ & 68.15 & 6.36 & 24.35 \\
M5 & 1 & $12: 5$ & 98.72 & 9.04 & 20.37 \\
M6 & 1 & $7: 5$ & 99.92 & 9.22 & 20.61 \\
M7 & 0.625 & $12: 5$ & 87.03 & 7.91 & 21.12 \\
M8 & 0.25 & $12: 5$ & 99.82 & 9.18 & 21.44 \\
M9 & 0.25 & $7: 5$ & 98.96 & 9.32 & 21.09 \\
\hline
\end{tabular}

Abbreviations: CCD, central composite design; RES, reticuloendothelial system; PI27, Pluronic 127; TPGS, D- $\alpha$-tocopheryl polyethylene glycol 1000 succinate; $\mathrm{EE}$, entrapment efficiency; DL, drug loading; PS, particle size.

dependent variables with a high correlation coefficient $(P<0.05, r=0.9133)$ were well described with quadratic polynomial equations, and the three-dimensional response surface plots were constructed according to regression equations with a design expert software.

As shown in Table 2, the EE and DL of resveratrol micelles ranged from $68.15 \%$ to $99.92 \%$ and $6.36 \%$ to $9.32 \%$, respectively. After statistical analysis, in this case, the results indicated that independent $\mathrm{B}$ is a significant model term affecting both EE and DL, with $P$-values of $<0.05$. The regression equations of the fitted model constructed for $\mathrm{EE}$ and DL are presented below:

$$
\begin{aligned}
Y_{1}(\mathrm{EE} \%)= & 98.81+0.65 A+8.71 B-1.46 A B \\
& -0.53 A^{2}-11.97 B^{2} \\
Y_{2}(\mathrm{DL} \%)= & 9.06+0.004 A+0.79 B-0.11 A B \\
& -0.01 A^{2}-1.13 B^{2}
\end{aligned}
$$

In Figure $3 \mathrm{~A}$ and $\mathrm{B}$, we observe that the influence of independent factors on $\mathrm{EE}$ and DL presents the similar tendency. Both of them changed slightly with the increase in the amount of resveratrol, which might be related to the fact that self-assembled P127/TPGS micelles could provide relatively settled spaces in configuration to accommodate moderate lipophilic drugs; therefore, the entrapment ability was mainly dependent on the number of micelles formed. Once the amount of drug fed exceeded the capability of the micelles, EE and DL remained stable. On the other hand, as the ratio of P127 to TPGS enhanced, the EE and DL gradually increased to their maximum point and then decreased like an umbrella asymptotic curve. This trend could be ascribed to
A

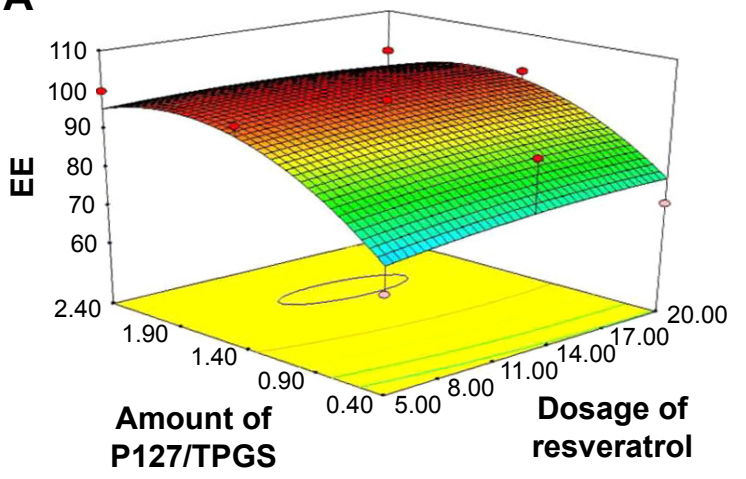

B

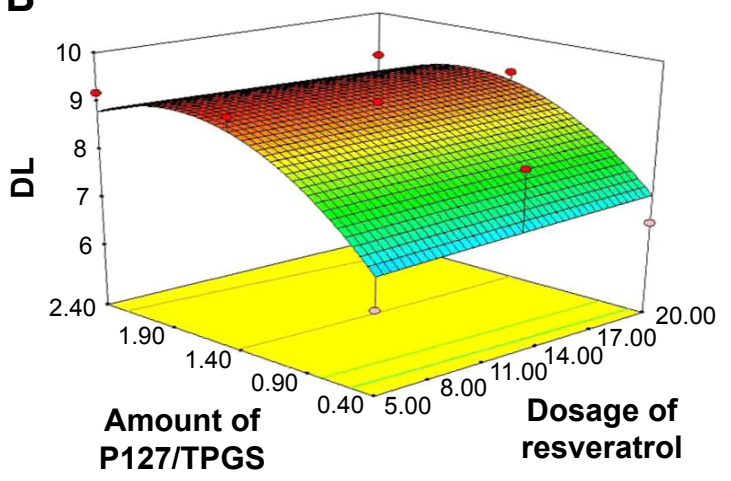

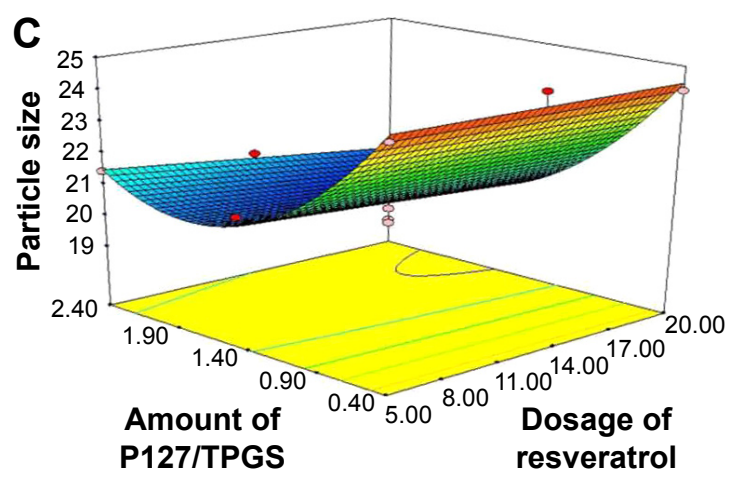

Figure 3 Response surface model showing the influence of the independent variables on the EE (A), DL (B) and particle size (C). Abbreviations: EE, encapsulation efficiency; PI27, Pluronic I27; TPGS, D- $\alpha$-tocopheryl polyethylene glycol I000 succinate; DL, drug loading. 
the increase in volume of the mixed micelles formed by the mosaic nonionic surfactant TPGS, which manifested synergistic properties to change and enlarge the dimension of the micelle cores to incorporate more drug species resulting in higher EE and DL. ${ }^{26}$

With regard to particle size, as we all know, particles of small size can escape clearance such as renal, hepatic and splenic filtration and exhibit prolonged blood circulation, which gives them an increased chance for long systemic circulation so as to achieve tumor accumulation. ${ }^{27}$ In addition, there is an increasing interest in fabrication of small particles exhibiting improved tumor permeability and penetration. ${ }^{28}$ Therefore, one goal of this study was to tailor colloidal micelles with a minimum particle size.

The data in Table 2 show that the mean particle sizes of mixed micelles ranged from 19.90 to $24.97 \mathrm{~nm}$. When particle size (Yps) was considered as the response, good correlation was found between observed and predicted value as revealed by an $r^{2}$ of 0.961 . The analysis of variance results revealed that in this case, independent $\mathrm{B}$ and polynomial term $B^{2}$ were significant model terms with $P$-values of $<0.05$. The following equation could explain the effect of factors level on particle size:

$$
\begin{aligned}
Y_{3}(\mathrm{PS})= & 20.56-0.28 A-1.77 B-0.24 A B \\
& -0.003 A^{2}+2.19 B^{2}
\end{aligned}
$$

As shown in Figure 3C, the particle size did not show pronounced variance with the enhanced amount of resveratrol, while as the ratio of P127/TPGS increased, trends of particle size presented in a saddle shape. This phenomenon could be attributed to the fact that particle size of the micelles merely depended on the type of nonionic surfactant. Once the micelles formed, the dimension of the micelles maintained a relatively stationary state. Thus, the quantity of drug had a slight influence on the particle size. Also, when TPGS was introduced as a mixed micelles matrix, Gibbs free energy of the dispersed system in water decreased to some degree as the surfactant concentration increased. Hence, the hydrophobic impact derived from a disorder between entropy and enthalpy of the micelles' dispersion could provide more driving forces for smaller size micelles formation.

After analyzing the dependent and independent factors, a further optimization and validation strategy by means of the design expert software was performed with desirable properties to optimize resveratrol-loaded micelles formula, which depended on the prescriptive criteria of maximum $\mathrm{EE}$ and DL and minimum particle size. The composition of optimum formulation was determined as $A=18.78 \mathrm{mg}$ (w/v, dosage of resveratrol) and $B=1.77$ (the amount of P127/ TPGS), which fulfilled the target requirements. At these levels, the predicted values of $Y_{\mathrm{EE}}, Y_{\mathrm{DL}}$ and $Y_{\mathrm{PS}}$ were $99.67 \%$, $9.15 \%$ and $19.9 \mathrm{~nm}$, respectively. Hence, in order to verify the predicted model, a new batch of micelles according to the optimal formulation factors level was prepared. The observed optimal formulation had an EE of $99.75 \% \pm 0.18 \%$, a DL of $9.39 \% \pm 0.11 \%$ and a particle size of $20.38 \pm 0.38 \mathrm{~nm}$, which was in good agreement with the predicted values. The low bias between these observed results and theoretical predictions indicates the reliability of CCD used in predicting the desirable micelles formulation.

\section{Physicochemical characterization of micelles}

Particle size and morphology

The optimum micelles for loading resveratrol were successfully prepared and then subjected to physicochemical characterization. The mean particle size of resveratrol mixed micelles was $\sim 19.81 \mathrm{~nm}$ with a polydispersity index of 0.098 as shown in Figure 4A. TEM image (Figure 4B) showed spherical and homogeneous mixed micelles, and the size correlated well with the results determined by laser diffraction method.

Particle size played a vital role in the absorption and distribution in vivo within the systemic circulation phase of drug delivery. The $\sim 20 \mathrm{~nm}$ size of polymeric micelles was smaller than the permeable vasculature pore diameter present in many solid tumors, suggesting that the prepared FA mixed micelles could be able to selectively accumulate in solid tumors by means of both the EPR effect and active targeting ability.

\section{CMC of resveratrol-loaded mixed micelles}

As nonionic surfactants, P127 and TPGS can spontaneously form micelles in aqueous circumstance once the concentration goes beyond the CMC. Pyrene fluorescent probe was generally adopted to determine the CMC value of mixed surfactants.

As shown in Figure 4C, the semilog plot of I372/I383 versus surfactant concentration was found to be sigmoidal in nature. Several parameters such as $A_{1}, A_{2}, x_{0}$ and $\Delta x$ can be directly read in figure, which were $0.82,0.71,-1.54$ and 0.18 , respectively. According to the literature, as long as the ratio of $x_{0} / \Delta x$ is $<10, x_{0}$ would be the required CMC value. ${ }^{29}$ Consequently, the final CMC value of P127/TPGS 
A

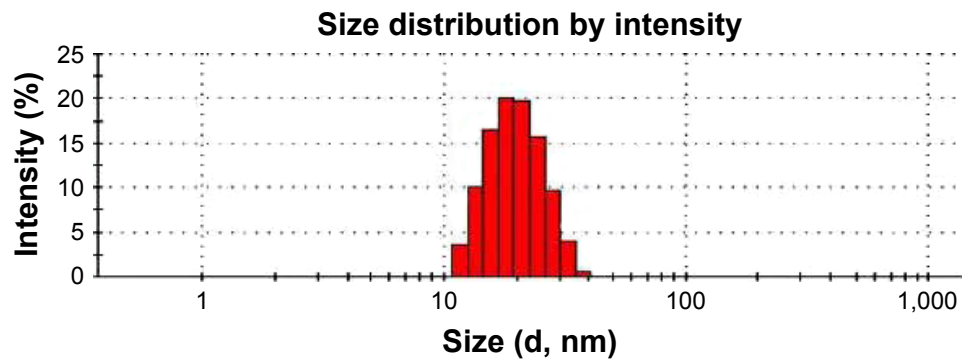

B

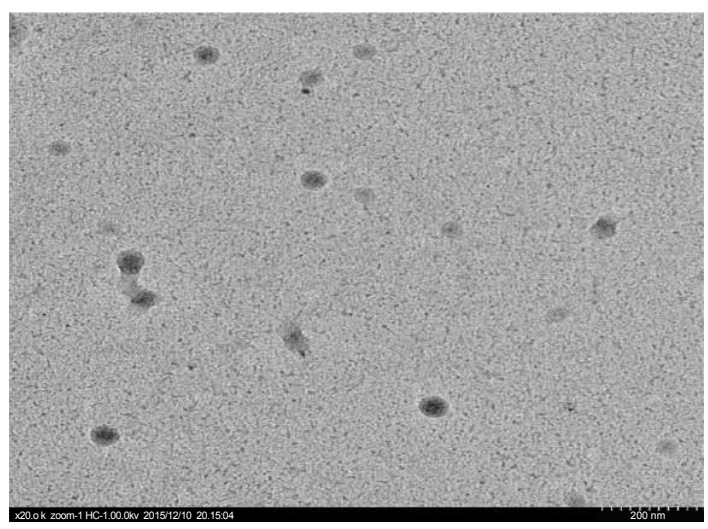

C

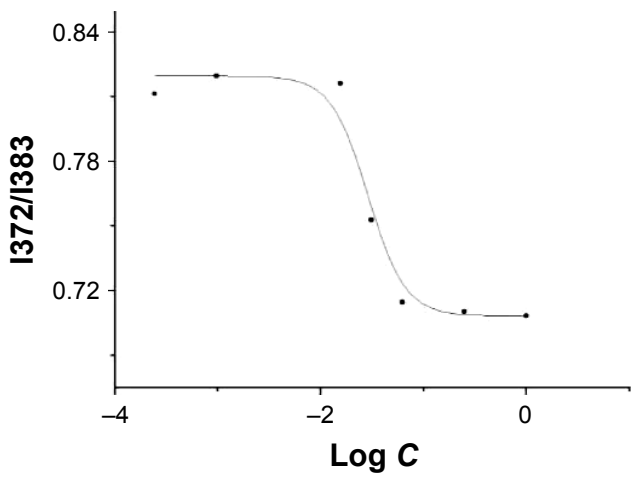

Figure 4 The physicochemical characteristics of FA-PI27/TPGS micelles: particle size and size distribution (A); TEM morphology images (B) and determination of CMC value of the micelles (C).

Abbreviations: FA, folic acid; PI27, Pluronic I27; TPGS, D- $\alpha$-tocopheryl polyethylene glycol 1000 succinate; TEM, transmission electron microscopy; CMC, critical micelle concentration.

mixed surfactant was $0.0288 \mathrm{mg} / \mathrm{mL}$. This relatively low $\mathrm{CMC}$ indicated that the mixed micelles had intensive thermodynamic stability. ${ }^{30,31}$

\section{Resveratrol release from mixed micelles in vitro}

The in vitro release of resveratrol from micelles under sink condition was performed by dialysis method with $0.5 \%$ Tween $80-$ PBS solution as release medium. As shown in Figure 5, within the first $2 \mathrm{~h}$, only $7.6 \%$ of resveratrol was released from the micelles, while $\sim 30 \%$ of resveratrol was released from the propylene glycol solution during the same time period. After $12 \mathrm{~h}$, the cumulative release of resveratrol from the propylene glycol solution reached up to $98.5 \%$; as for the cumulative release from micelles is concerned, the liberation of only $23 \%$ of drug suggested that $\sim 77 \%$ of the initially incorporated drug was still present in the micelles. The results meant a sustained-release behavior of the micelles, which might be ascribed to the maze-escape of the drug from the core-shell configuration due to which the drug-release period was prolonged. The release of resveratrol from micelles may be attributed to drug diffusion or the polymer material erosion and swelling. ${ }^{32,33}$

\section{In vitro cell evaluation}

\section{Cytotoxicity analysis}

CCK-8-based in vitro cytotoxicity evaluation of free and resveratrol-loaded micelles was performed using MCF-7 cells (FA-positive receptor). Figure 6A exhibits the results of cell viability obtained using MCF-7 cells after exposure for $48 \mathrm{~h}$. The blank FA-P127/TPGS micelles did not show

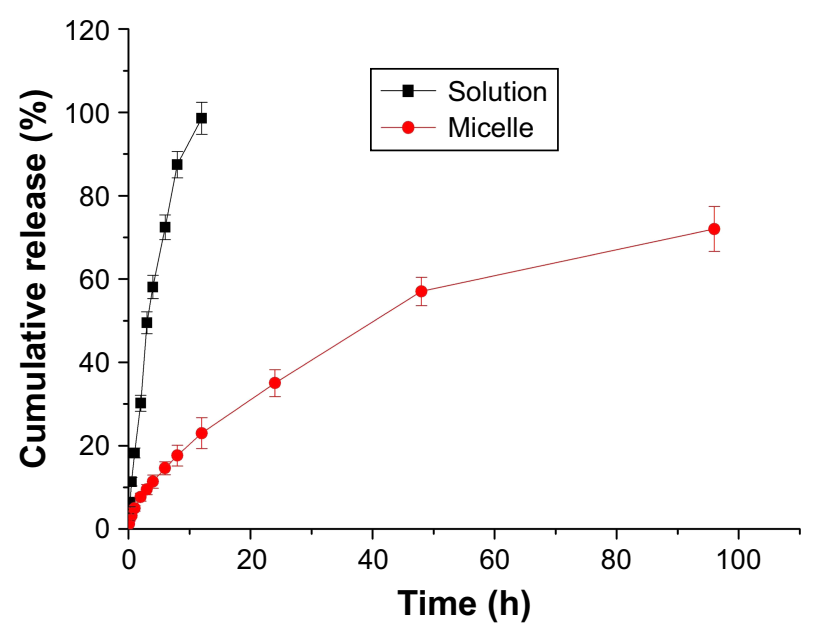

Figure 5 Profiles of the cumulative release percentage of resveratrol from mixed micelles and the polyethylene glycol solution. 


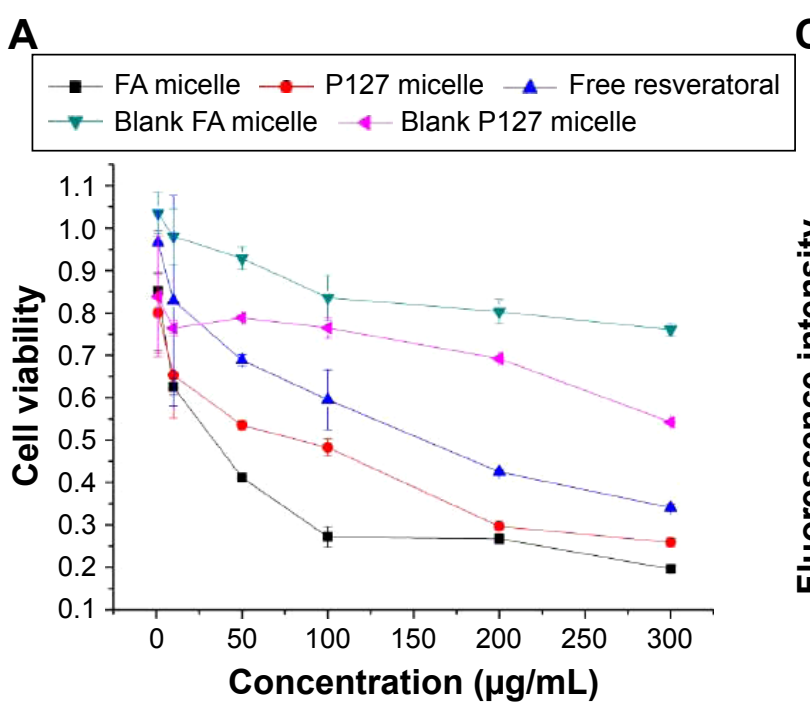

\section{C}

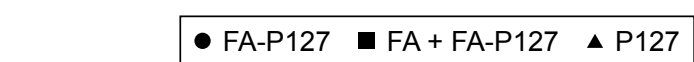

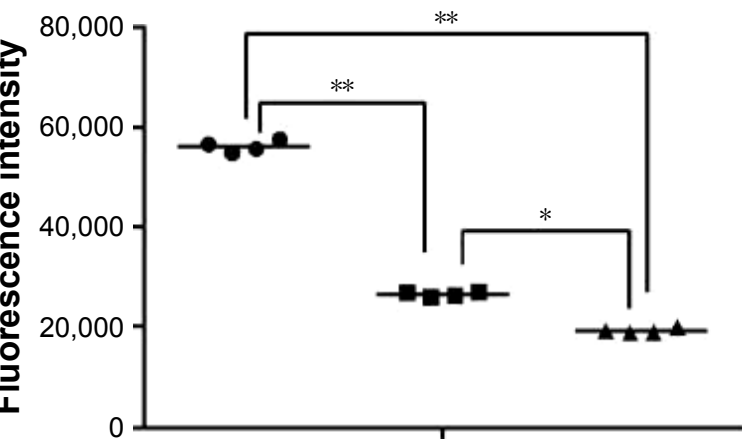

B

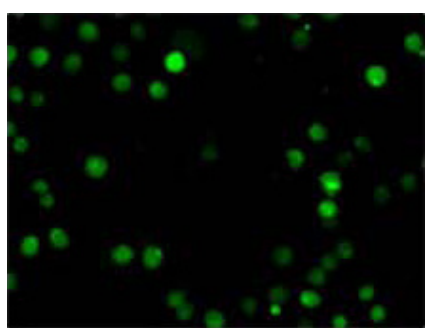

P127

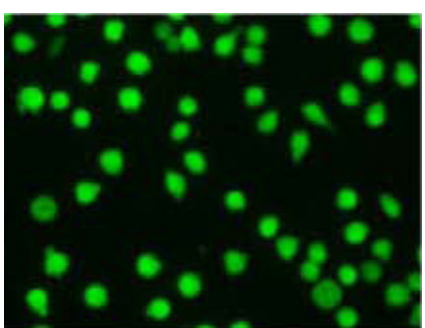

FA-P127

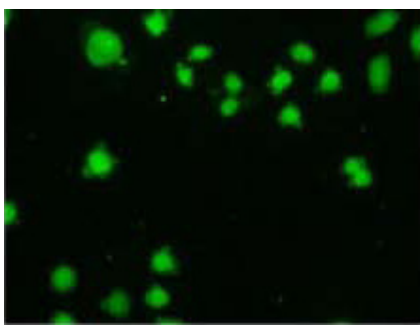

FA + FA-P127

Figure 6 In vitro cell line evaluation: in vitro cytotoxicity of micelles in MCF-7 cells (A); fluorescence microscopic images of micelles internalized (B) and cell uptake efficiency of micelles (C). $* P<0.05, * * P<0.01$.

Abbreviations: FA, folic acid; PI27, Pluronic 127.

any significant cytotoxicity against MCF-7 cells, while the blank P127/TPGS micelles displayed some harm to MCF-7 cells at relatively higher concentration. This may be attributed to the fact that excessive surfactant consisted in the micelles will jeopardize various proteins and phospholipid membranes when contacting with cells, which increase the permeability of cell membranes and cause leakage of low-molecular mass compounds resulting in cell death or damage.

The $\mathrm{IC}_{50}$ values for FA micelles, F407 and resveratrol were $27.89,35.19$ and $137.58 \mu \mathrm{g} / \mathrm{mL}$, respectively, suggesting the superior cytotoxicity of folate-mediated micelles. The degree of cell viability (Figure 6A) further disclosed that resveratrol-loaded FA-targeted micelles are more cytotoxic than free drug and nonmodified micelles against MCF-7 cells especially in lower concentration, probably because the presence of FA on the micelles surface incurred more uptake of resveratrol-loaded FA-targeted micelles by the MCF-7 cells via receptor-mediated endocytosis. ${ }^{34}$

\section{In vitro cellular uptake of micelles}

To further probe the selectivity of FA micelles internalized into MCF-7 cells, a competition study in the presence of
FA was performed to confirm whether the cellular uptake involved an FAR-mediated mechanism. For this, $1 \mathrm{mM}$ free FA-containing DMEM was incubated in advance with MCF-7 cells for $30 \mathrm{~min}$ before adding FA micelles. The fluorescence microscopic technique was utilized to evaluate the cellular uptake of FITC-loaded FA-P127 micelles and P127 micelles. As shown in Figure 6B, the fluorescence image demonstrated that both FA-P127 micelles and P127 micelles were internalized into the MCF-7 cells as shown by the green fluorescence in the cytoplasm after incubation for $2 \mathrm{~h}$. Also, the fluorescence intensity was determined by a microplate reader (Tecan Infinite M1000 PRO; Tecan Group Ltd., Männedorf, Switzerland), and the results are shown in Figure 6C. Comparing the fluorescence intensity of FA-P127 micelles with that of P127 micelles and FA blocking control, it could be understood that FA-P127 micelles with the FA molecules indeed had the stronger intensity. This could be explained by the fact that the free FA molecules occupying the folate receptors on MCF-7 cell membranes hinder the cellular internalization of FA-P127 into MCF-7 cells. ${ }^{35}$ The results of the experiment conducted to investigate the competition between free FA and FA-P127 specifically showed that 

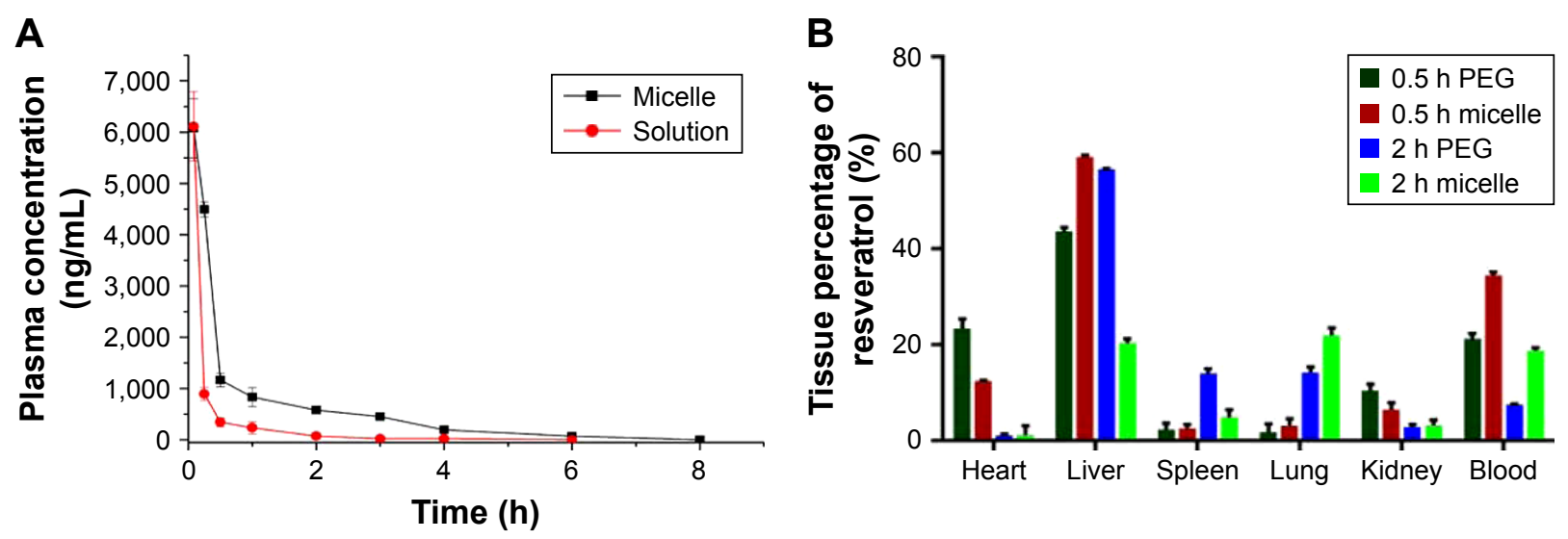

Figure 7 Plasma concentration versus time profile of resveratrol after i.v. administration (A) and biodistribution of resveratrol after i.v. administration (B). Abbreviations: i.v., intravenous; PEG, polyethylene glycol.

more micelles were absorbed via the specific uptake pathway mediated by FAR than by nonspecific uptake. ${ }^{17}$

\section{In vivo pharmacokinetic study}

The plasma concentration versus time curves of resveratrol after intravenous (i.v.) administration of two formulations in rats are shown in Figure 7A. The results implied that resveratrol solution showed rapid clearance from the circulation system in comparison with that of the micelles. On the contrary, administration of resveratrol micelles showed extended residence of resveratrol in systemic blood circulation. This phenomenon could be explained as follows: 1) The presence of three phenolic hydroxyl groups in the chemical configuration facilitated resveratrol to act as a good substrate for hepatic sulfotransferase and glucuronosyl transferase, thus leading to a swift clearance, while the encapsulation of drug into the micelles' internal cavity provided an effective shield against the metabolism, accordingly increasing the plasma concentration. 2) The hydrophilic shell of three block polymers formed micelles with "core-shell" configuration that could confer the micelles the potential to avoid opsonization and prevent rapid clearance by the mononuclear phagocyte system, resulting in the high blood concentration profiles compared with the control. Also, statistical moment analysis was used to evaluate the pharmacokinetic properties. Mean pharmacokinetic parameters for the two preparations are shown in Table 3. It can be observed that the AUC value of resveratrol micelles was 2.73-fold higher in comparison with that of the control solution. Moreover, the MRT of resveratrol micelles was significantly longer than that of resveratrol solution. It was deduced that micelles could improve the availability of resveratrol by the slow release of drug from the micelles and evasion of clearance. ${ }^{36}$
Resveratrol concentration in different tissues was determined to assess its distribution upon i.v. administration of resveratrol solution and micelles in mice. Figure $7 \mathrm{~B}$ presents the biodistribution data of resveratrol FA micelles and resveratrol solution at 0.5 and $2 \mathrm{~h}$ after i.v. administration. At $0.5 \mathrm{~h}$, resveratrol accumulated in the heart, liver and kidney, with a lesser amount in the spleen and lung. But at the same time interval, more distribution in the liver was observed from micelles than that of solution. This increased uptake could be attributed to the reticuloendothelial system leading to an increase in resveratrol concentration in the liver. It is generally accepted that the clearance and tissue distribution of either macroparticulate or nanoparticulate drug carriers administered intravenously are greatly affected by their size, surface features and opsonization. Upon entering the blood, systemically injected drugs and particles are tagged with proteins and subsequently removed by the mononuclear phagocyte system. ${ }^{37}$ Therefore, a higher concentration of drug is accumulated in the organ-resident mononuclear phagocyte system. At $2 \mathrm{~h}$, resveratrol solution group exhibited decreased concentration in the heart and kidney as evaluated in this study, while micelles concentration in the organs such as the spleen and

Table 3 Pharmacokinetic parameters of resveratrol micelles and the reference after i.v. administration $(n=5)$

\begin{tabular}{lll}
\hline Parameter & Micelles & Solutions \\
\hline AUC $_{0 \rightarrow \infty}(\mathrm{mg} / \mathrm{L} \cdot \mathrm{h})$ & $4,683.46 \pm 351.26^{*}$ & $1,716.30 \pm 310.22$ \\
$\mathrm{AUMC}_{0 \rightarrow \infty}$ & $5,739.42 \pm 278.34^{*}$ & $763.5 \mathrm{I} \pm 262.7 \mathrm{I}$ \\
$\mathrm{CL}(\mathrm{L} / \mathrm{h} / \mathrm{kg})$ & 0.005 & 0.013 \\
$\mathrm{MRT}(\mathrm{h})$ & $1.23 \pm 0.07$ & $0.42 \pm 0.08$ \\
\hline
\end{tabular}

Notes: Statistical significance compared with reference. $* P<0.05$. Data are represented as the mean \pm SD.

Abbreviations: i.v., intravenous; $\mathrm{AUC}_{0 \rightarrow \infty}$, area under the plasma concentrationtime curve from zero to infinity; $A \cup M C_{0 \rightarrow \infty}$, area under the plasma concentration versus times curve (zero moment) and the first moment curve; $C L$, clearance; MRT, mean residence time; SD, standard deviation. 
lung increased in comparison with that at $0.5 \mathrm{~h}$. This could be explained by the fact that in some permeable tissues, the nanoparticles can move to the interstitial perisinusoidal space of the organs through the capillary systems and then may be intercepted by the lung and spleen after i.v. administration, which enables them to accumulate in tissues. ${ }^{38}$ In addition, a significantly higher concentration in plasma was observed in micelles group at the different interval when compared to the PEG solution group. This may be due to the presence of a long hydrophilic chain of P127 in the FA micelles, which results in increased circulation time in the plasma. ${ }^{39}$

\section{Conclusion}

In this study, a functional resveratrol-loaded mixed micelles platform based on FA-P127 and TPGS was developed through thin-film dispersion approach. As a result of the formulation optimization, the micelles exhibited spherical shape, small size and high DL and EE. Compared with the propylene glycol solution, the micelles showed a sustained-release behavior, which increased their circulation time. The mixed micelles exhibited enhanced cell uptake via FAR-mediated endocytosis. Pharmacokinetic and biodistribution experiments disclosed the increased AUC and MRT and decreased resveratrol accumulation in the heart and kidney, which may avoid toxicity to these vital organs. Thus, the functional mixed micelles may be considered as a promising delivery platform for resveratrol.

\section{Acknowledgments}

This work was supported by the grants obtained from Natural Science Foundation of Shandong Province (Nos ZR2016HM21, ZR2014HL103, J13LM51) and Taishan Medical University Foundation (No 2014GCC05).

\section{Disclosure}

The authors report no conflicts of interest in this work.

\section{References}

1. Amri A, Chaumeil JC, Sfar S, Charrueau C. Administration of resveratrol: what formulation solutions to bioavailability limitations? J Control Release. 2012;158(2):182-193.

2. Carletto B, Berton J, Ferreira TN, et al. Resveratrol-loaded nanocapsules inhibit murine melanoma tumor growth. Colloid Surf B Biointerfaces. 2016;144:65-72.

3. Wu Z, Liu B, Cailing E, et al. Resveratrol inhibits the proliferation of human melanoma cells by inducing G1/S cell cycle arrest and apoptosis. Mol Med Rep. 2015;11(1):400-404.

4. Summerlin N, Soo E, Thakur S, Qu Z, Jambhrunkar S, Popat A. Resveratrol nanoformulations: challenges and opportunities. Int J Pharm. 2015;479(2):282-290.

5. Pujara N, Jambhrunkar S, Wong KY, McGuckin M, Popat A. Enhanced colloidal stability, solubility and rapid dissolution of resveratrol by nanocomplexation with soy protein isolate. J Colloid Interface Sci. 2017;488:303-308.
6. Jeon YO, Lee JS, Lee HG. Improving solubility, stability, and cellular uptake of resveratrol by nanoencapsulation with chitosan and $\gamma$-poly (glutamic acid). Colloids Surf B Biointerfaces. 2016;147:224-233.

7. Cosco D, Paolino D, Maiuolo J, et al. Ultradeformable liposomes as multidrug carrier of resveratrol and 5-fluorouracil for their topical delivery. Int J Pharm. 2015;489(1-2):1-10.

8. Paolino D, Cosco D, Gaspari M, et al. Targeting the thyroid gland with thyroid-stimulating hormone (TSH)-nanoliposomes. Biomaterials. 2014;35(25):7101-7109.

9. Paolino D, Cosco D, Licciardi M, Giammona G, Fresta M, Cavallaro G. Polyaspartylhydrazide copolymer-based supramolecular vesicular aggregates as delivery devices for anticancer drugs. Biomacromolecules. 2008;9(4):1117-1130.

10. Paolino D, Celia C, Trapasso E, Cilurzo F, Fresta M. Paclitaxelloaded ethosomes ${ }^{\circledR}$ : potential treatment of squamous cell carcinoma, a malignant transformation of actinic keratoses. Eur J Pharm Biopharm. 2012;81(1):102-112.

11. El-Dahmy RM, Elsayed I, Elshafeey AH, Gawad NA, El-Gazayerly ON. Optimization of long circulating mixed polymeric micelles containing vinpocetine using simple lattice mixture design, in vitro and in vivo characterization. Int J Pharm. 2014;477(1-2):39-46.

12. Dong L, XiaS, WuK, etal. A pH/enzyme-responsive tumor-specific delivery system for doxorubicin. Biomaterials. 2010;31(24):6309-6316.

13. Canal F, Vicent MJ, Pasut G, Schiavon O. Relevance of folic acid/ polymer ratio in targeted PEG-epirubicin conjugates. J Control Release. 2010;146(3):388-399.

14. Li W, Szoka FC Jr. Lipid-based nanoparticles for nucleic acid delivery. Pharm Res. 2007;24(3):438-449.

15. Kim SH, Jeong JH, Chun KW, Park TG. Target-specific cellular uptake of PLGA nanoparticles coated with poly(L-lysine)-poly(ethylene glycol)-folate conjugate. Langmuir. 2005;21(19):8852-8857.

16. Liu L, Yong KT, Roy I, et al. Bioconjugated pluronic triblock-copolymer micelle-encapsulated quantum dots for targeted imaging of cancer: in vitro and in vivo studies. Theranostics. 2012;2(7):705-713.

17. Zhang D, Tao L, Zhao H, Yuan H, Lan M. A functional drug delivery platform for targeting and imaging cancer cells based on Pluronic F127. J Biomater Sci Polym Ed. 2015;26(8):468-482.

18. Fang J, Nakamura $H$, Maeda $H$. The EPR effect: unique features of tumor blood vessels for drug delivery, factors involved, and limitations and augmentation of the effect. Adv Drug Deliv Rev. 2011;63(3):136-151.

19. Zhang W, Shi Y, Chen Y, Ye J, Sha X, Fang X. Multifunctional Pluronic P123/F127 mixed polymeric micelles loaded with paclitaxel for the treatment of multidrug resistant tumors. Biomaterials. 2011; 32(11):2894-2906.

20. Wei Z, Hao J, Yuan S, et al. Paclitaxel-loaded Pluronic P123/F127 mixed polymeric micelles: formulation, optimization and in vitro characterization. Int J Pharm. 2009;376(1-2):176-185.

21. Jansson J, Schillén K, Olofsson G, da Silva RC, Loh W. The interaction between PEO-PPO-PEO triblock copolymers and ionic surfactants in aqueous solution studied using light scattering and calorimetry. $J$ Phys Chem B. 2004;108(1):82-92.

22. Basu Ray G, Chakraborty I, Moulik SP. Pyrene absorption can be a convenient method for probing critical micellar concentration $(\mathrm{cmc})$ and indexing micellar polarity. J Colloid Interface Sci. 2006;294(1): 248-254.

23. Lin JJ, Chen JS, Huang SJ, et al. Folic acid-Pluronic F127 magnetic nanoparticle clusters for combined targeting, diagnosis, and therapy applications. Biomaterials. 2009;30(28):5114-5124.

24. Huang C, Tang Z, Zhou Y, et al. Magnetic micelles as a potential platform for dual targeted drug delivery in cancer therapy. Int J Pharm. 2012;429(1-2):113-122.

25. Bagalkot V, Farokhzad OC, Langer R, Jon S. An aptamer-doxorubicin physical conjugate as a novel targeted drug-delivery platform. Angew Chem Int Ed Engl. 2006;45(48):8149-8152.

26. Xu W, Cui Y, Ling P, Li LB. Preparation and evaluation of folatemodified cationic pluronic micelles for poorly soluble anticancer drug. Drug Deliv. 2012;19(4):208-219. 
27. Liu CH, Wu CT. Optimization of nanostructured lipid carriers for lutein delivery. Colloids Surf A. 2010;353(2-3):149-156.

28. Ernsting MJ, Murakami M, Roy A, Li SD. Factors controlling the pharmacokinetics, biodistribution and intratumoral penetration of nanoparticles. J Control Release. 2013;172(3):782-794.

29. Aguiar J, Carpena P, Molina-BolíVar JA, Carnero Ruiz C. On the determination of the critical micelle concentration by the pyrene 1:3 ratio method. J Colloid Interface Sci. 2003;258(1):116-122.

30. Chen W, Miao YQ, Fan DJ, et al. Bioavailability study of berberine and the enhancing effects of TPGS on intestinal absorption in rats. AAPS PharmSciTech. 2011;12(2):705-711.

31. Duan Y, Cai X, Du H, Zhai G. Novel in situ gel systems based on P123/TPGS mixed micelles and gellan gum for ophthalmic delivery of curcumin. Colloids Surf B Biointerfaces. 2015;128:322-330.

32. Kim KS, Park SJ. Effect of porous silica on sustained release behaviors of $\mathrm{pH}$ sensitive pluronic F127/poly(acrylic acid) hydrogels containing tulobuterol. Colloids Surf B Biointerfaces. 2010;80(2):240-246.

33. Li N, Yang X, Zhai G, Li L. Multifunctional pluronic/poly(ethylenimine) nanoparticles for anticancer drug. J Colloid Interface Sci. 2010;350(1): $117-125$.
34. Wang Y, Yu L, Han L, Sha X, Fang X. Difunctional Pluronic copolymer micelles for paclitaxel delivery: synergistic effect of folate-mediated targeting and Pluronic-mediated overcoming multidrug resistance in tumor cell lines. Int J Pharm. 2007;337(1-2):63-73.

35. Zhou Q, Guo X, Chen T, et al. Target-specific cellular uptake of folatedecorated biodegradable polymer micelles. J Phys Chem B. 2011; 115(43):12662-12670.

36. Pawar H, Surapaneni SK, Tikoo K, et al. Folic acid functionalized long-circulating co-encapsulated docetaxel and curcumin solid lipid nanoparticles: in vitro evaluation, pharmacokinetic and biodistribution in rats. Drug Deliv. 2016;23(4):1453-1468.

37. Parodi A, Quattrocchi N, van de Ven AL, et al. Synthetic nanoparticles functionalized with biomimetic leukocyte membranes possess cell-like functions. Nat Nanotechnol. 2013;8(1):61-68.

38. Jia L, Zhang D, Li Z, et al. Nanostructured lipid carriers for parenteral delivery of silybin: biodistribution and pharmacokinetic studies. Colloids Surf B Biointerfaces. 2010;80(2):213-218.

39. Deng J, Huang L, Liu F. Extraction issues of paclitaxel in nanocrystals. J Biomed Nanotechnol. 2010;6(2):198-201.
International Journal of Nanomedicine

\section{Publish your work in this journal}

The International Journal of Nanomedicine is an international, peerreviewed journal focusing on the application of nanotechnology in diagnostics, therapeutics, and drug delivery systems throughout the biomedical field. This journal is indexed on PubMed Central,

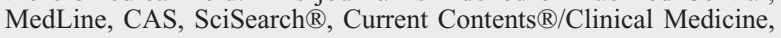

\section{Dovepress}

Journal Citation Reports/Science Edition, EMBase, Scopus and the Elsevier Bibliographic databases. The manuscript management system is completely online and includes a very quick and fair peer-review system, which is all easy to use. Visit http://www.dovepress.com/ testimonials.php to read real quotes from published authors. 